\title{
Pregnancy Outcomes After Endometrioma Excision in Patients Undergoing In Vitro Fertilization and Embryo Transfer: A Historical Cohort Study
}

\author{
Rubin Raju, MD, Komal Agarwal, MD, Omar Abuzeid, MD, ${ }^{1}$ Salem Joseph, BS, \\ Mohammed Ashraf, MD, ${ }^{1-3}$ and Mostafa I. Abuzeid, MD ${ }^{1-3}$
}

\begin{abstract}
Objective: The objective of the study was to examine the effect of endometrioma excision on pregnancy outcomes in women with advanced-stage endometriosis who underwent in vitro fertilization and embryo transfer (IVF-ET). Design: This is a historical cohort study. Materials and Methods: We compared the pregnancy outcomes of 141 women undergoing IVF-ET. The study group consisted of 25 patients who had stage III/IV endometriosis and endometrioma excision (group 1). The control groups included 40 patients who had stage III/ IV endometriosis, but no endometrioma and who underwent ovariolysis (group 2) and 76 patients with tubal factors infertility who underwent tubal surgery (group 3). After surgery up to two IVF-ET cycles in each group were analyzed. Results: Our study showed that the mean total dose of gonadotropin administered in IVF-ET cycle I was higher in group 1 compared with groups 2 and $3(p=0.03)$. Otherwise, there was no significant difference in the ovarian responses among the three groups. There was a statistically significant increase in clinical pregnancy rate per cycle in the endometrioma group $(69.7 \%)$ versus the ovariolysis group (48.1\%) and tubal factor group (48.0\%). However, there was no significant difference in delivery rate per cycle among the three groups. There was a statistically significant higher miscarriage rate in the endometrioma group (39.1\%) compared with the ovariolysis group (11.5\%) and tubal factor group (14.3\%). Conclusion: In conclusion, our study suggests that endometrioma excision surgery does not compromise the overall ovarian reserve or pregnancy outcomes after IVF-ET. (J GYNECOL SURG 31:214)
\end{abstract}

\section{Introduction}

$\mathbf{E}$ NDOMETRIOSIS IS A BENIGN DISEASE characterized by the presence of endometrial glands and stroma outside the uterus. Its overall prevalence among reproductive age women is estimated to be between $3 \%$ and $10 \%$, but it affects between $20 \%$ and $40 \%$ of infertile women. ${ }^{1}$ Endometriomas affect $17 \%-$ $44 \%$ of women with endometriosis. ${ }^{2}$ The origin of ovarian endometrioma is unknown; however, it is generally believed that they result initially from a deposit of endometrium passed through the fallopian tube, causing adherence of the ovary to the pelvic peritoneum and progressive invagination of the ovarian cortex. ${ }^{3-5}$

Endometriomas are associated with increased oxidative stress and vascular compromise that can cause damage to ovarian reserve and function. ${ }^{6}$ Controversy exists with respect to their management. Surgical resection of endometriomas is widely practiced to overcome infertility, due to poor response to medical therapy, but this practice is not yet supported by solid evidence. ${ }^{6}$ A risk of surgical removal of ovarian endometrioma is the inadvertent loss of normal ovarian tissue. ${ }^{7}$ There are studies that suggest that excision of endometriomas is associated with a reduction in antimüllerian hormone (AMH) levels, suggestive of a decrease in ovarian reserve, which can have significant implications on fertility outcomes. ${ }^{8-10}$

The purpose of this study was to examine the effect of endometrioma excision on pregnancy outcomes in women with advanced-stage endometriosis underdoing in vitro fertilization and embryo transfer (IVF-ET).

\footnotetext{
${ }^{1}$ Department of Obstetrics and Gynecology, Michigan State University College of Human Medicine, Flint Campus, Flint, MI.

${ }^{2}$ IVF Michigan PC, Rochester Hills, MI.

${ }^{3}$ Division of Reproductive Endocrinology and Infertility, Department of Obstetrics and Gynecology, Michigan State University College of Human Medicine, Flint Campus, Flint, MI.

Presented as an abstract at the American Association of Gynecologic Laparoscopists (AAGL) 38th Global Congress of Minimally Invasive Gynecology. Orlando, Florida, November 15-19, 2009, S16, O-51.
} 


\section{Materials and Methods}

This is a historical cohort study using a retrospective chart review and a database from the years 1991 to 2009 of infertile patients who presented to our unit. The study was approved by the Institutional Review Board at Hurley Medical Center, Michigan Flint.

A total of 141 patients were included in our study. The inclusion criteria for the study group (group 1) were infertile patients undergoing IVF-ET with stage III/IV endometriosis and a history of unilateral or bilateral laparoscopic endometrioma excision $(n=25)$. The diagnosis of endometrioma was histologically confirmed. The control groups included patients who had stage III/IV endometriosis but no endometrioma who underwent ovariolysis and (group 2, $n=40$ ) and patients with tubal factors infertility who underwent tubal surgery (group $3, n=76$ ). Group 3 consisted of patients with no laparoscopic evidence of endometriosis who underwent tubal surgery for tubal factor infertility, failed to conceive, and, subsequently, underwent IVF-ET.

The operative laparoscopy and IVF-ET were performed by the same group of infertility specialists (Mostafa I. Abuzeid and Mohammed Ashraf) under similar operative setting in all patients. The same surgical techniques were applied in all patients. When suspicious spots of endometriosis were found anywhere in the pelvis, in the majority of cases, argon beam coagulator (Birtcher Medical System, Irvine, CA) was used to ablate the spots seen. If endometriosis was found on the pelvic sidewall near the ureter, on the bladder or bowels, Argon beam coagulator was also used with extra care to avoid injury to such vital organs. Salpingo-ovariolysis was performed using blunt dissection of the ovary from the pelvic sidewall, and scissors and/or monopolar diathermy needle tip (Elmed, Chicago, IL) and Teflon probe (Elmed) to excise the scar tissue from the tubes and ovaries. If endometrioma more than $1 \mathrm{~cm}$ was found, excision of the cyst wall was done and reconstruction of the ovarian cortex was performed with one or two interrupted sutures using 3-0 Vicryl. ${ }^{11}$ If an endometrioma of less than $1 \mathrm{~cm}$ was found, it was opened, evacuated, and the lining was ablated with the argon beam coagulator. If fimbrial phimosis or hydrosalpinges were found, fimbrioplasty or salpingostomy was performed, respectively, as previously described by our group. ${ }^{12}$ Starting in 1999 , temporary ovarian suspension was performed for advanced endometriosis in an attempt to reduce the risk of recurrence of adhesions between ovarian fossa and ovaries as previously described by our group. ${ }^{13}$

All patients underwent controlled ovarian stimulation (COS) using mid luteal downregulation protocol with GnRH-agonist or antagonist protocol and gonadotropin. The starting dose of gonadotropin was determined based on a woman's age, body mass index, antral follicle count at baseline trans-vaginal ultrasonography, and day 3 follicle-stimulating hormone (FSH). ${ }^{14}$ Ovarian response was monitored with frequent serum E2 levels and trans-vaginal ultrasonography.

The criterion for human chorionic gonadotropin (HCG) administration was the presence of three or more follicles that were $17 \mathrm{~mm}$ in diameter. Cycle cancellation due to poor ovarian response was undertaken when less than three follicles developed. Oocyte retrieval was carried out under modified general anesthesia using trans-vaginal ultrasound (US) guided puncture of follicles at 36 hours after HCG administration. Standard procedures were carried out for the gamete-embryo handling, and ET was performed in all cases using the Wallace catheter. In the period between 1991 and 2001, ET was performed on day 3 after oocyte retrieval. During the last 7 years, ET was performed on day 5 .

Embryos were graded on day 2 based on blastomere nuclear scoring ${ }^{14}$ and their morphologic appearance on day 3 cleavage embryos. ${ }^{15}$ On day 3 , if six to eight embryos were of good quality, the transfer was delayed until day 5; otherwise, the embryos were transferred on day 3. Blastocysts were graded according to Gardner's criteria. ${ }^{16}$ Ultrasound-guided ET was performed on either day 3 or 5. Usually, two top-quality embryos/blastocysts were transferred. Extra good-quality blastocysts were frozen. Luteal phase support was the same in all groups. Over the years, different forms of vaginal progesterone were used; however, the principal remained the same. Starting on the second day after retrieval: Progesterone vaginal suppositories T.I.D., progesterone vaginal cream once a day (Crinone 8\% Vaginal Gel; Watson Pharmaceuticals, Morristown, NJ) or progesterone vaginal tablets T.I.D. (Endometrin $100 \mathrm{mg}$ Vaginal Insert; Ferring Pharmaceuticals, Inc., Parsippany, NJ) and progesterone in oil $100 \mathrm{mg}$ I.M. every other day (Progesterone in Oil $50 \mathrm{mg} / \mathrm{mL}$ Vial; Watson Laboratories, Inc., Corona, CA) were used. Since the year 2005, Estradiol B.I.D. (Estrace $2 \mathrm{mg}$; Warner Chilcott LLC, Rockaway, NJ) were also utilized for luteal phase support. If pregnancy occurred, the same treatment continued until 6 week of gestation. At that time, Estrace and Progesterone in Oil were discontinued, and vaginal progesterone was continued until 12 week of gestation.

Pregnancy was confirmed by measurement of $\beta$-HCG at 12 days after blastocyst transfer, or 14 days if ET was done on day 3. Biochemical pregnancy was defined as a transit rise in $\beta$-HCG, or a positive pregnancy test in the absence of US scan evidence of pregnancy. Clinical pregnancy was defined as the presence of a gestational sac on trans-vaginal US scan at 6 week of gestation. Miscarriage was defined as a clinical pregnancy that ended in pregnancy loss before 12 weeks of gestation. Delivery was verified by a phone call to the patient.

Up to two IVF-ET cycles after surgery were analyzed. The outcomes studied were as follows: pregnancy rate per cycle, pregnancy rate per patient, delivery rate per cycle, delivery rate per patient, and miscarriage rate. Statistical analyses were performed using Student's $t$-test, chi-square test (continuous and dichotomous variables, respectively),

Table 1. Demographic Data

\begin{tabular}{lccc}
\hline & $\begin{array}{c}\text { Endometrioma } \\
\text { excision, } \\
\text { group 1 } \\
(\mathrm{n}=25)\end{array}$ & $\begin{array}{c}\text { Ovariolysis, } \\
\text { group 2 } \\
(\mathrm{n}=40)\end{array}$ & $\begin{array}{c}\text { Tubal } \\
\text { group, } \\
\text { group 3 } \\
(\mathrm{n}=76)\end{array}$ \\
\hline Age & $\begin{array}{r}34.3 \pm 4.1 \\
2.6 \pm 1.7\end{array}$ & $\begin{array}{r}33.1 \pm 3.9 \\
3.7 \pm 3.4\end{array}$ & $\begin{array}{r}32.1 \pm 3.2 \\
4.0 \pm 3.5\end{array}$ \\
$\begin{array}{l}\text { Duration } \\
\text { of infertility }\end{array}$ & 68 & 59 & 49.4 \\
$\begin{array}{l}\text { Primary } \\
\text { infertility (\%) }\end{array}$ & 28 & 17.5 & 17.3 \\
\hline \begin{tabular}{l} 
Septal surgery $(\%)$ \\
\hline
\end{tabular}
\end{tabular}


TABle 2. VARiables IN IVF-ET CyCle I

\begin{tabular}{lccc}
\hline Variables & $\begin{array}{c}\text { Endometrioma excision, } \\
\text { group } 1(\mathrm{n}=25)\end{array}$ & $\begin{array}{c}\text { Ovariolysis } \\
\text { group, 2 }(\mathrm{n}=40)\end{array}$ & $\begin{array}{c}\text { Tubal group, } \\
\text { group 3 }(\mathrm{n}=76)\end{array}$ \\
\hline Days till HCG & $10.4 \pm 1.6$ & $11.0 \pm 1.4$ & $11.0 \pm 1.9$ \\
Total dose of gonadotropin administered (I.U.)* & $3825.0 \pm 1473.5$ & $3101.6 \pm 1185.1$ & $3071.8 \pm 1269.6$ \\
No. of mature follicles & $8.5 \pm 4.4$ & $10.3 \pm 5.6$ & $9.8 \pm 4.9$ \\
Estrogen level (pg/mL) & $2062.4 \pm 813.3$ & $2230.0 \pm 1068.5$ & $2072.9 \pm 1102.7$ \\
Progesterone level (ng/mL) & $0.9 \pm 0.4$ & $1.1 \pm 0.6$ & $1.0 \pm 0.8$ \\
No. of mature eggs & $9.2 \pm 4.4$ & $10.0 \pm 5.5$ & $10.7 \pm 5.7$ \\
No. of fertilized eggs & $7.5 \pm 4.4$ & $7.6 \pm 4.7$ & $8.6 \pm 5.4$ \\
No. of embryos transferred & $3.2 \pm 1.2$ & $3.1 \pm 1.3$ & $3.0 \pm 1.3$ \\
\hline
\end{tabular}

$* p=0.03$.

HCG, human chorionic gonadotropin; IVF-ET, in vitro fertilization and embryo transfer.

and one-way analysis of variance; these were used where appropriate, considering a $p$-value $<0.05$ as statistically significant.

\section{Results}

Group mean age, mean duration of infertility, type of infertility, and history of septal surgery were not different between the three groups, as shown in Table 1. Table 2 illustrates ovarian stimulation characteristics for the patients undergoing IVF-ET during the first cycle. The mean total medicine administered in IVF-ET cycle I in group 1, the patients who underwent endometrioma excision were higher $(p=0.03)$. Apart from this, there was no significant difference among the three groups with respect to the number of treatment days, E2 levels, progesterone levels, number of mature follicles on $\mathrm{HCG}$ day, number of mature eggs, number of fertilized eggs, and number of embryos transferred in IVF-ET cycle I. Patients who did not conceive or miscarried after the first IVF-ET cycle or whose cycle were cancelled underwent a second IVF-ET cycle. Table 3 illustrates ovarian stimulation characteristics for the patients undergoing the second IVF-ET cycle. There was no significant difference among the three groups with respect to the number of treatment days, total dose of gonadotropins administered, E2 levels, progesterone levels, number of mature follicles on HCG day, number of mature eggs, number of fertilized eggs, and number of embryos transferred in IVF-ET cycle II. Combined pregnancy outcomes following the IVF-ET cycles I and II are shown in Table 4. The clinical pregnancy rate per cycle was higher in group 1 compared with groups 2 and $3(69.7 \%$ vs. $48 \%$ and $51 \%$ respectively; $p$-value $<0.01)$.
Miscarriage rate was significantly higher in group 1 compared with groups 2 and 3 (39.1\% vs. $11.5 \%$ and $14.3 \%$ respectively; $p$-value $<0.003)$. There was no significant difference in the delivery rate per cycle and delivery rate per patient among the three groups (Table 4).

\section{Discussion}

The appropriate management of endometriomas in women suffering from subfertility is still a topic of debate. Several surgical techniques have been described. Most involve opening and draining the cyst followed by either excision using the stripping technique or ablation of the cyst wall with electrocautery or $\mathrm{CO}_{2}$ laser. ${ }^{17}$ Due to a high recurrence rate, drainage alone is not recommended. ${ }^{17} \mathrm{~A}$ Cochrane review found that excisional surgery provides better outcomes than ablative surgery. ${ }^{18}$ Donnez et al. (2010) described a combined technique using both excision and ablation to minimize ovary damage and recurrence rates. ${ }^{19}$

The main concern with surgery is the associated inadvertent removal of ovarian parenchyma that can have a significant impact on ovarian reserve. Muzii et al. showed that the stripping technique removes ovarian tissue in about $54 \%$ of cases. ${ }^{20} \mathrm{~A}$ significant reduction in ovarian reserve has also been demonstrated after endometrioma excision as assessed by decreased AMH levels and antral follicle counts. ${ }^{8-10} \mathrm{Bu}-$ sacca et al. reported $2.4 \%$ rate of premature ovarian failure immediately after bilateral endometrioma surgery. ${ }^{21}$ Such a report is alarming, since bilateral lesions account for 19\%$28 \%$ in patients with ovarian endometriosis. ${ }^{22}$ Coccia et al. have also shown that in women previously subjected to bilateral endometrioma removal, the mean age at menopause is

TABle 3. Variables in IVF-ET CyCle II

\begin{tabular}{lccc}
\hline Variables & $\begin{array}{c}\text { Endometrioma excision, } \\
\text { group } 1(\mathrm{n}=8)\end{array}$ & $\begin{array}{c}\text { Ovariolysis, } \\
\text { group 2 }(\mathrm{n}=14)\end{array}$ & $\begin{array}{c}\text { Tubal group, } \\
\text { group 3 }\end{array}$ \\
\hline Days till HCG & $10.5 \pm 1.4$ & $10.5 \pm 1.5$ & $10.9 \pm 1.85$ \\
Total medication administered & $4219 \pm 548$ & $3081.7 \pm 1299$ & $3305 \pm 1431$ \\
No. of mature follicles & $7 \pm 2.2$ & $10.7 \pm 6.5$ & $9.15 \pm 3.8$ \\
Estrogen level & $1834 \pm 103.1$ & $2282.5 \pm 1173$ & $2175.4 \pm 968$ \\
Progesterone level & $1.0 \pm 0.4$ & $1.0 \pm 0.8$ & $1.2 \pm 0.6$ \\
No. of mature eggs & $8.6 \pm 3.7$ & $10.5 \pm 6.9$ & $10 \pm 4.3$ \\
No. of fertilized eggs & $6.0 \pm 1.6$ & $8.5 \pm 6.3$ & $8.0 \pm 4.1$ \\
No. of embryos transferred & $3.5 \pm 1.1$ & $3.7 \pm 1.7$ & $3.3 \pm 1.7$ \\
\hline
\end{tabular}


Table 4. Combined Pregnancy Outcomes After IVF-ET Cycles I and II

\begin{tabular}{|c|c|c|c|}
\hline & $\begin{array}{l}\text { Endometrioma excision, } \\
\text { group } 1(\mathrm{~N}=25)\end{array}$ & $\begin{array}{l}\text { Ovariolysis, } \\
\text { group } 2(\mathrm{~N}=40)\end{array}$ & $\begin{array}{c}\text { Tubal, group } 3 \\
(\mathrm{~N}=76)\end{array}$ \\
\hline No. of IVF-ET cycles & 33 & 54 & 102 \\
\hline No. of clinical pregnancies & 23 & 26 & 49 \\
\hline Clinical pregnancy rate per cycle $(\%)^{*}$ & 69.7 & 48.1 & 48.0 \\
\hline No. of patients delivered & 14 & 23 & 42 \\
\hline Delivery rate per cycle $(\%)$ & 42.2 & 42.6 & 41.2 \\
\hline Delivery rate per patient $(\%)$ & 56.0 & 57.5 & 55.3 \\
\hline No. of miscarriages $(\%)^{* *}$ & $9(39.1)$ & $3(11.5)$ & $7(14.3)$ \\
\hline
\end{tabular}

significantly lower. ${ }^{23}$ Factors shown to influence ovarian reserve after surgery are the expertise of the surgeon, the size of the cyst, and the composition of the cyst wall. ${ }^{10,24} \mathrm{Yu}$ et al. showed a significantly diminished antral follicle count in the hands of an inexperienced surgeon for both unilateral and bilateral cystectomies. ${ }^{10}$ Romualdi et al. showed that the smaller cysts and fibroblastic capsular types were more associated with the follicles being removed during surgery, likely due to less demarcated cleavage plane. ${ }^{24}$ In such patients, de-roofing and ablation is the preferred procedure to minimize follicular loss.

The effect of ovarian surgery on ovarian response to fertility medications is subject to a matter of debate. Criteria for poor ovarian response include small numbers of developed follicles or retrieved oocytes, and low estradiol (E2) levels. $^{25}$ Our study showed no difference in the overall ovarian responses among the three populations studied. In two of such populations studied (group 2 and group 3), excision of endometrioma was not performed. Therefore, our data suggest that excision of endometrioma does not affect ovarian response to fertility medications. Similarly, Canis et al. in a retrospective study showed that in the hands of an experienced surgeon there was no significant reduction in the number of oocytes retrieved. ${ }^{25}$ In addition, in a retrospective study of women with severe endometriosis who underwent laparoscopic vaporization of the cyst wall, Donnez et al. showed that in women with unilateral endometriomas undergoing excision there was a similar ovarian response in cystectomized ovaries when compared with the contralateral nonoperated ovary. ${ }^{26}$ In a Cochrane review in 2008, Hart et al. showed an increased ovarian response with excisional procedures than with ablative procedures for endometrioma removal in women undergoing IVF. ${ }^{18}$ On the other hand, several retrospective studies have reported reduced responses to gonadotrophins after endometrioma excision. ${ }^{27-31}$ Such discrepancy in the literature can be related to the experience of the surgeon and other surgical factors such as the nature of cyst, the baseline ovarian reserve in some patients, and surgical technique as mentioned earlier.

Our study showed that the mean total dose of gonadotropin administered in IVF-ET cycle I was higher $(p=0.03)$ in patients who underwent endometrioma excision. This could be related to an element of decrease ovarian reserve secondary to surgery and is consistent with existing studies. However, such differences were not seen in the second cycle, probably due to the smaller sample size (group 1: $n=8$, group 2: $n=14$ and group 3: $n=26$ ). Similarly, other investigators have reported higher gonadotrophin dose after endometrioma excision surgery in women undergoing IVF. ${ }^{32-34}$

Our study showed a statistically significant increase in pregnancy rate per cycle in the endometrioma group (69.7\%) versus in the ovariolysis group $(48.1 \%)$ and tubal factor group (48.0\%). The delivery rates per cycle and per patient were not significantly different among the three groups. This could be explained by the statistically significant increase in miscarriage rate in the endometrioma group $(39.1 \%)$ versus the ovariolysis group $(11.5 \%)$ and tubal factors group (14.3\%). Similar to our overall results, In a retrospective study of women with severe endometriosis who underwent laparoscopic vaporization of the cyst wall, Donnez et al. showed similar IVF outcomes when compared with women having tubal infertility. ${ }^{26}$ However, the effect of ovarian surgery on pregnancy rates in women undergoing IVF-ET is still a matter of debate. In a randomized controlled trial, Demirol et al.showed that ovarian surgery in women undergoing IVF treatment did not affect the fertilization, implantation, and pregnancy rates. ${ }^{33}$ Similarly, GarciaVelasco et al. showed that previous endometrioma excision surgery in women undergoing IVF did not improve the number of oocytes retrieved, number of embryos obtained/transferred, and the pregnancy rates. ${ }^{34,35} \mathrm{Yu}$ et al. showed better live birth rates when experienced laparoscopic surgeons treated endometriomas in women undergoing IVF. ${ }^{10}$ In a Cochrane review, Hart et al.showed that removal of endometriomas were associated with a significant increase of spontaneous pregnancy in subfertile women. ${ }^{18}$

The association between endometriosis and an increased risk of spontaneous miscarriage is also a topic of debate. There have been retrospective studies in the past that have shown an increased risk of pregnancy loss with endometriosis. ${ }^{36-38}$ These studies lacked control groups. More recent studies have refuted this and shown no causal relationship. ${ }^{39,40}$ Our study showed an increased miscarriage rate in the endometrioma excision group in comparison to the ovariolysis group, although patients in both groups had stage III/VI endometriosis. In addition, our data suggest higher miscarriage rate in the endometrioma excision group compared with the tubal factor group. More research in needed to evaluate the possible association between miscarriage and some patients with endometriosis, especially after IVF treatment. 
Our study has some limitations as a result of its retrospective nature with the inherent biases that are associated with such design and its sample size, which may limit its generalizability. One area of strength is there were no significant differences in age, duration of infertility, or the type of infertility between the three populations studied. Another area of strength in our study is the fact that all surgeries were performed using the same technique. In addition, during IVFET, all COS and ETs were performed by only two physicians (Mostafa I. Abuzeid and Mohammed Ashraf), which limit confounding factors that may affect implantation rates and, in turn, pregnancy outcomes. Our study compared the study group (patients who had endometrioma excision) with another group of patients with the same stage of endometriosis except that they had no endometrioma. This may have controlled for some confounding factors related to endometriosis, whereas most existing studies have only tubal factor infertility as the control group.

\section{Conclusion}

Our study suggests that excision of endometrioma in patients with advanced endometriosis before IVF-ET treatment does not compromise the overall ovarian reserve or pregnancy outcomes. Large, prospective, and randomized controlled trials are still required to shed more light on this matter.

\section{Acknowledgments}

The authors would like to acknowledge the Hurley Research Department for helping them with the statistical analysis of their data. They would also like to acknowledge Cheryl Anderson for helping them prepare this article.

\section{Disclosure Statement}

No competing financial interests exist.

\section{References}

1. Fritz MA, Speroff L. Clinical Gynecologic Endocrinology and Infertility, 8th ed. Philadelphia: Lippincott Williams \& Wilkins, 2011.

2. Vercellini P, Chapron C, De Giorgi O, Consonni D, Frontino G, Crosignani PG. Coagulation or excision of ovarian endometriomas? Am J Obstet Gynecol 2003;188:606.

3. Brosens IA, Puttemans PJ, Deprest J. The endoscopic localization of endometrial implants in the ovarian chocolate cyst. Fertil Steril 1994;61:1034.

4. Brosens IA, Van Ballaer P, Puttemans P, Deprest J. Reconstruction of the ovary containing large endometriomas by an extraovarian endosurgical technique. Fertil Steril 1996;66:517.

5. Nisolle M. Ovarian endometriosis and peritoneal endometriosis: Are they different entities from a fertility perspective? Curr Opin Obstet Gynecol 2002;14:283.

6. Ruiz-Floresa F, Garcia-Velascoa J. Is there a benefit for surgery in endometrioma associated infertility? Curr Opin Obstet Gynecol 2012;24:136.

7. Wattiez A, Puga M, Albornoz J, Faller E. Surgical strategy in endometriosis. Best Pract Res Clin Obstet Gynaecol 2013;27:381.

8. Somigliana E, Berlanda N, Benaglia L, Viganò P, Vercellini $\mathrm{P}$, Fedele L. Surgical excision of endometriomas and ovarian reserve: A systematic review on serum antimüllerian hormone level modifications. Fertil Steril 2012;98:1531.

9. Raffi F, Metwally M, Amer S. The impact of excision of ovarian endometrioma on ovarian reserve: A systematic review and meta-analysis. J Clin Endocrinol Metab 2012; 97:3146.

10. Yu HT, Huang HY, Soong YK, Lee CY, Chao A, Wang CJ. Laparoscopic ovarian cystectomy of endometriomas: Surgeons' experience may affect ovarian reserve and liveborn rate in infertile patients with in vitro fertilizationintracytoplasmic sperm injection. Eur J Obstet Gynecol Reprod Biol 2010;152:172.

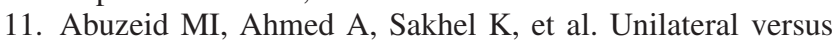
bilateral adnexal disease in stage III and stage IV endometriosis does not affect pregnancy outcome after operative laparoscopy. Obstet Gynecol Surv 2009;64:452.

12. Mitwally M, Thotakura A, Abuzeid O, Ashraf M, Diamond M, Abuzeid MI. Suturing versus flowering technique of Bruhat after fimbrioplasty for endometriosis-related infertility. Gynecol Surg 2009;6:147.

13. Abuzeid MI, Ashraf M, Shamma FN. Temporary ovarian suspension at laparoscopy for prevention of adhesions. J Am Assoc Gynecol Laparosc 2002;9:98.

14. Van Royen E, Mangelschots K, Vercruyssen M, De Neubourg D, Valkenburg M, Ryckaert G, Gerris J. Multinucleation in cleavage stage embryos. Hum Reprod 2003; 18:1062-1069.

15. Khan I, Staessen C, Devroey P, Van Steirteghem A. Human serum albumin versus serum: a comparative study on embryo transfer medium. Fertil Steril 1991;56:98-101.

16. Gardner D, Surrey E, Minjarez D, Leitz A, Stevens J, Schoolcraft W. Single blastocyst transfer: a prospective randomized trial. Fertil Steril 2004;81:551-555.

17. Holoch KJ, Shenoy CC, Falcone T. Surgical management of endometriosis. Expert Rev Obstet Gynecol 2013;8:475.

18. Hart RJ, Hickey M, Maouris P, et al. Excisional surgery versus ablative surgery for ovarian endometriomata (Review). Cochrane Database Syst Rev 2008;4:1.

19. Donnez J, Lousse JC, Jadoul P, Donnez O, Squifflet J. Laparoscopic management of endometriomas using a combined technique of excisional (cystectomy) and ablative surgery. Fertil Steril 2010; 94:28-32.

20. Muzii L, Bianchi A, Croce C, Manci N, Panici PB. Laparoscopic excision of ovarian cysts: Is the stripping technique a tissue-sparing procedure? Fertil Steril 2002; 77:609.

21. Busacca M, Riparini J, Somigliana E, et al. Postsurgical ovarian failure after laparoscopic excision of bilateral endometriomas. Am J Obstet Gynecol 2006;95:421.

22. Somigliana E, Arnoldi M, Benaglia L, Iemmello R, Nicolosi AE, Ragni G. IVF-ICSI outcome in women operated on for bilateral endometriomas. Hum Reprod 2008;23: 1526.

23. Coccia ME, Rizzello F, Mariani G, et al. Ovarian surgery for bilateral endometriomas influences age at menopause. Hum Reprod 2011;26:3000.

24. Romualdi D, Franco Zannoni G, Lanzone A, et al. Follicular loss in endoscopic surgery for ovarian endometriosis: Quantitative and qualitative observations. Fertil Steril 2011;96:374.

25. Canis M, Pouly JL, Tamburro S, et al. Ovarian response during IVF-embryo transfer cycles after laparoscopic ovarian cystectomy for endometriotic cysts of $>3 \mathrm{~cm}$ in diameter. Hum Reprod 2001;16:2583. 
26. Donnez J, Wyns C, Nisolle M. Does ovarian surgery for endometriomas impair the ovarian response to gonadotropin? Fertil Steril 2001;76:662.

27. Pagidas K, Falcone T, Hemmings R, Miron P. Comparison of reoperation for moderate (stage III) and severe (stage IV) endometriosis-related infertility with in vitro fertilizationembryo transfer. Fertil Steril 1996;65:791.

28. Loh FH, Tan AT, Kumar J, Ng SC. Ovarian response after laparoscopic ovarian cystectomy for endometriotic cysts in 132 monitored cycles. Fertil Steril 1999;72:316.

29. Al-Azemi M, Bernal AL, Steele J, Gramsbergen I, Barlow D, Kennedy S. Ovarian response to repeated controlled stimulation in in-vitro fertilization cycles in patients with ovarian endometriosis. Hum Reprod 2000;15:72.

30. Tinkanen $\mathrm{H}$, Kujansuu $\mathrm{E}$. In vitro fertilization in patients with ovarian endometriomas. Acta Obstet Gynecol Scand 2000;79:119.

31. Ragni G, Somigliana E, Benedetti F, et al. Damage to ovarian reserve associated with laparoscopic excision of endometriomas: A quantitative rather than a qualitative injury. Am J Obstet Gynecol 2005;193:1908.

32. Marconi G, Vilela M, Quintana R, Sueldo C. Laparoscopic ovarian cystectomy of endometriomas does not affect the ovarian response to gonadotropin stimulation. Fertil Steril 2002;78:876.

33. Demirol A, Guven S, Baykal C, Gurgan T. Effect of endometrioma cystectomy on IVF outcome: A prospective randomized study. Reprod Biomed Online 2006;12:639.

34. Garcia-Velasco JA, Mahutte NG, Corona J, et al. Removal of endometriomas before in vitro fertilization doesnot im- prove fertility outcomes: A matched, case--control study. Fertil Steril 2004;81:1194.

35. Garcia-Velasco JA, Somigliana E. Management of endometriomas in women requiring IVF: To touch or not to touch. Hum Reprod 2009;24:496.

36. Naples JD, Batt RE, Sadigh H. Spontaneous abortion rate in patients with endometriosis. Obstet Gynecol 1981;57:509.

37. Wheeler JM, Johnston BM, Malinak LR. The relationship of endometriosis to spontaneous abortion. Fertil Steril 1983;39:656.

38. Groll M. Endometriosis and spontaneous abortion. Fertil Steril 1984;44:933.

39. Vercammen EE, D'Hooghe TM. Endometriosis and recurrent pregnancy loss. Semin Reprod Med 2000;18:363.

40. Matalliotakis I, Cakmak H, Dermitzaki D, Zervoudis S, Goumenou A, Fragouli Y. Increased rate of endometriosis and spontaneous abortion in an in vitro fertilization program: No correlation with epidemiological factors. Gynecol Endocrinol 2008;24:194.

Address correspondence to:

Rubin Raju, $M D$

Department of Obstetrics and Gynecology

Hurley Medical Center

Michigan State University

College of Human Medicine

Flint Campus

Flint, MI 48503

E-mail:rraju1@hurleymc.com 